\title{
Mutational spectrum of the ED1 gene in X-linked hypohidrotic ectodermal dysplasia
}

\author{
Marie C Vincent ${ }^{1}$, Valérie Biancalana ${ }^{2}$, Danièle Ginisty ${ }^{3}$, Jean L Mandel ${ }^{2,4}$ and \\ Patrick Calvas*,1
}

\begin{abstract}
${ }^{1}$ Service de Génétique Médicale, Hôpital Purpan CHU Toulouse, Pavillon Lefebvre, 1 place Baylac, 31059 Toulouse Cedex, France; ${ }^{2}$ Laboratoire de Diagnostic Génétique, Faculté de médecine CHRU Strasbourg, 11 rue Human, 67085 Strasbourg Cedex, France; ${ }^{3}$ Service de Stomatologie Pédiatrique, Groupe Hospitalier Saint Vincent de PaulLa Roche Guyon Assistance Publique de Paris, 74-82 avenue Denfert Rochereau, 75674 Paris Cedex 14, France; ${ }^{4}$ Institut de Génétique et Biologie Moléculaire et Cellulaire, CNRS/INSERM/Université Louis Pasteur de Strasbourg, BP 163, 67404 Illkirch Cedex, France
\end{abstract}

X-linked hypohidrotic ectodermal dysplasia (XLHED) is the most common form of the ectodermal dysplasias characterised by an abnormal development of eccrine sweat glands, hair and teeth. The ED1 gene responsible for the disorder undergoes extensive alternative splicing and to date few studies have concerned the full length transcript. We screened $\mathbf{5 2}$ unrelated families or sporadic cases for mutation in the full coding sequence of this gene. SSCA analysis or direct sequencing allowed identification of mutations in 34 families: one initiation defect, twenty-two missenses, two nonsense, eight insertions or deletions, and a large deletion encompassing all the ED1 gene. Fourteen of these mutations have not been previously described, including five missenses. One third of identified mutations were localised in codons 155 and 156, affecting CpG dinucleotides and nine of them correspond to the R156H missense. Hypothesis of a founder effect has been ruled out by haplotype analysis of flanking microsatellites. These recurrent mutations indicate the functional importance of the positively charged domain of the protein. Including our data, there are now 56 different mutations reported in $\mathbf{8 5}$ independent patients, that we have tabulated. Review of clinical features in the present series of affected males and female carriers showed no obvious correlation between the type of mutations, the phenotype and its severity. The X-chromosome pattern of inactivation in leucocytes showed little correlation with expressivity of the disease in female carriers. Finally this study is useful for functional studies of the protein and to define a diagnostic strategy for mutation screening of the ED1 gene. European Journal of Human Genetics (2001) 9, 355-363.

Keywords: mutational spectrum; ED1 gene; ectodysplasin; phenotype/genotype correlation; recurrent mutation

Introduction

Hypohidrotic ectodermal dysplasia (HED) is found worldwide with an estimated incidence of 1 per 100000 births. The X-linked hypohidrotic ectodermal dysplasia (XLHED: MIM

${ }^{*}$ Correspondence: P Calvas, Service de Génétique Médicale, Hôpital Purpan CHU Toulouse, Pavillon Lefebvre, 1 place Baylac, 31059 Toulouse cedex, France.

Tel: +33 5617790 79; Fax: +33 561779073

E-mail: calvas.p@chu-toulouse.fr

Received 1 September 2000; revised 18 January 2001; accepted 23 January 2001
305100) is the most common form of the HED characterised by an abnormal development of eccrine sweat glands, hair, and teeth. Affected males present most or all of the typical features of HED. ${ }^{1,2}$ Heterozygous carriers of XLHED may show minor to moderate degrees of these typical features. About 30\% do not present obvious clinical manifestations, rendering accurate diagnosis of carrier status in females difficult. $^{3-5}$

The gene (ED1) for XLHED, maps to Xq12-q13 ${ }^{6-9}$ and has been identified. ${ }^{3,10,11}$ So mutation analysis enables diagnosis in sporadic cases and carrier detection in female relatives avoiding the difficulties of genetic counselling due to the 
rarer, autosomal form of the disorder (AHED), which is clinically indistinguishable from XLHED, in affected males. ${ }^{1,3,5,12,13}$

The ED1 gene undergoes extensive alternative splicing. This generates several transcripts: the initially described shortest EDA-O form (two exons encoding a 135 aminoacid protein, mutated in 5-10\% of XLHED patients), the longest EDA-A form (nine exons, 391 aminoacids) and five others forms. ${ }^{10,11,14}$ All the transcripts share exon 1 which encodes a short intracellular domain, a single transmembrane domain and an extracellular domain of 66 aminoacids. In addition, the longest 391 aminoacid EDA-A form, includes a positively charged domain (arginine and lysine rich), a 19 repeats Gly$\mathrm{X}-\mathrm{Y}$ collagenous domain, a TNF-like domain and a cysteinerich C-terminal domain. ${ }^{3,11,14}$

Structure prediction indicates that the ED1 polypeptide, named Ectodysplasin, which is a new member in the TNFrelated ligand family, belongs to the class II transmembrane proteins and forms trimers. ${ }^{11,14,15}$ The collagenous, TNF-like and cysteine-rich domains are needed for trimer formation, contact and stability. In addition the TNF-like and cysteinerich domains are involved in proper processing of the protein. ${ }^{14}$

Mutations in the ED1 gene have been previouly reported

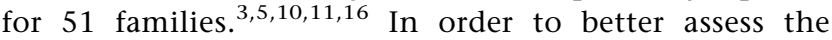
mutation spectrum, we performed mutation screening in 52 unrelated families or sporadic XLHED cases. Mutations were found in 34 of them (65\%) including 14 novel ones. A review of clinical features in this series of affected males and carrier females aimed to evaluate genotype/phenotype correlations. Combined with previous mutation reports, this study allowed us to define a diagnostic strategy for mutations screening of the ED1 gene, in order to improve genetic counselling.

\section{Materials and methods Patients}

Thirty-four affected males and eight females carries from 42 unrelated families and 10 sporadic cases with presumed XLHED, were screened for mutations of the ED1 gene after we obtained their informed consent according to the french law.

\section{Mutations screening}

Genomic DNA was prepared from venous blood according to standard protocols. The nine exons of the ED1 gene with intronic flanking sequences were amplified by PCR using previously published primers. ${ }^{10,11}$

Thermocycling was carried out as described by Bayes et al, ${ }^{11}$ except that the annealing temperature for exons 7 and 8 was $58^{\circ} \mathrm{C}$. For SSCA, denaturated PCR products were separated on a non denaturing MDE gel (TEBU, FMC Bioproducts-Europe, Denmark), run at $4{ }^{\circ} \mathrm{C}$ under two conditions: overnight at $3 \mathrm{~W}$ and $4-5 \mathrm{~h}$ at $12 \mathrm{~W}$ (constant power) and then visualised by silver staining. ${ }^{17}$ For sequencing, PCR fragments were subsequently purified with QIAquick Gel Extraction (QIAGEN SA, France) and sequenced using the Perkin-Elmer dyeterminator cycle sequencing kit (PE Biosystem, UK). Reactions were analysed in an ABI373Strecht sequencer (PE Biosystem, UK) and sequence analysis was carried out using Mac Vector programs (version 6.5, Oxford Molecular Genetic, UK).

Whenever possible, some mutations were screened by restriction enzyme digestion of PCR-amplified exon DNAs, using specific enzymes as the $\mathrm{R} 156 \mathrm{H}$ and the R244X mutations abolish respectively a Fnu4HI and a XhoI restriction site.

In order to detect deletions of ED1 gene in patient with XLHED, a single multiplex polymerase chain reaction (PCR) was designed. PCR were performed with a set of primer pairs for exons 3, 6, 7, 8 and 9 of ED1 gene. An internal control consisted in simultaneous amplification of the STR from the human androgen receptor gene. Cycling conditions were

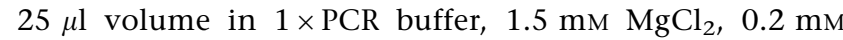
dNTPs, $0.2 \mu \mathrm{M}$ of each primer, 0.5 unit Goldstar Taq DNA Polymerase (Eurogentec, SA France) and 200 ng genomic DNA. Samples were submitted to 38 cycles of denaturation at $94^{\circ} \mathrm{C}$ for $30^{\prime \prime}$, annealing at $59^{\circ} \mathrm{C}$ for $1^{\prime}$ and extension at $72^{\circ} \mathrm{C}$ for $2^{\prime}$. PCR products were separated in composite $1.5 \%$ Nusieve $+1 \%$ Agarose gels.

\section{Microsatellite genotyping}

Polymorphic markers DXS1689, DXS1690 and DXS339 closely flanking the ED1 gene ${ }^{8,12}$ and two intragenic polymorphisms (IVS4+27T $>$ A and IVS5+8delCC) were used in order to define haplotypes in patients from different extraction bearing identical mutations.

\section{Pattern of $\mathrm{X}$ inactivation}

$\mathrm{X}$ inactivation patterns were analysed as described ${ }^{18}$ from 20 mutated carrier females, to compare the ratio of inactivation of the chromosome bearing the mutation in peripheral blood DNA. PhosphorImager 445SI scanning instrument (Molecular dynamic) was used to detect and quantify radioactivity for each X chromosome.

\section{Results}

\section{Mutational spectrum}

Screening of the entire coding sequence of the ED1 gene by SSCA and direct sequencing was performed in the 52 independent patients allowing identification of 34 mutated alleles, including 22 distinct mutations (Figure 1). Fourteen of these are novel mutations (indicated in bold in Tables 1 and 2).

The majority (22/34) of detected mutations were missense changes. Eleven mutations predict an absent or truncated protein (seven frameshifts, including an exon 3 deletion, two nonsense mutations, one translation initiation mutation, and the first reported complete deletion of the ED1 gene). 


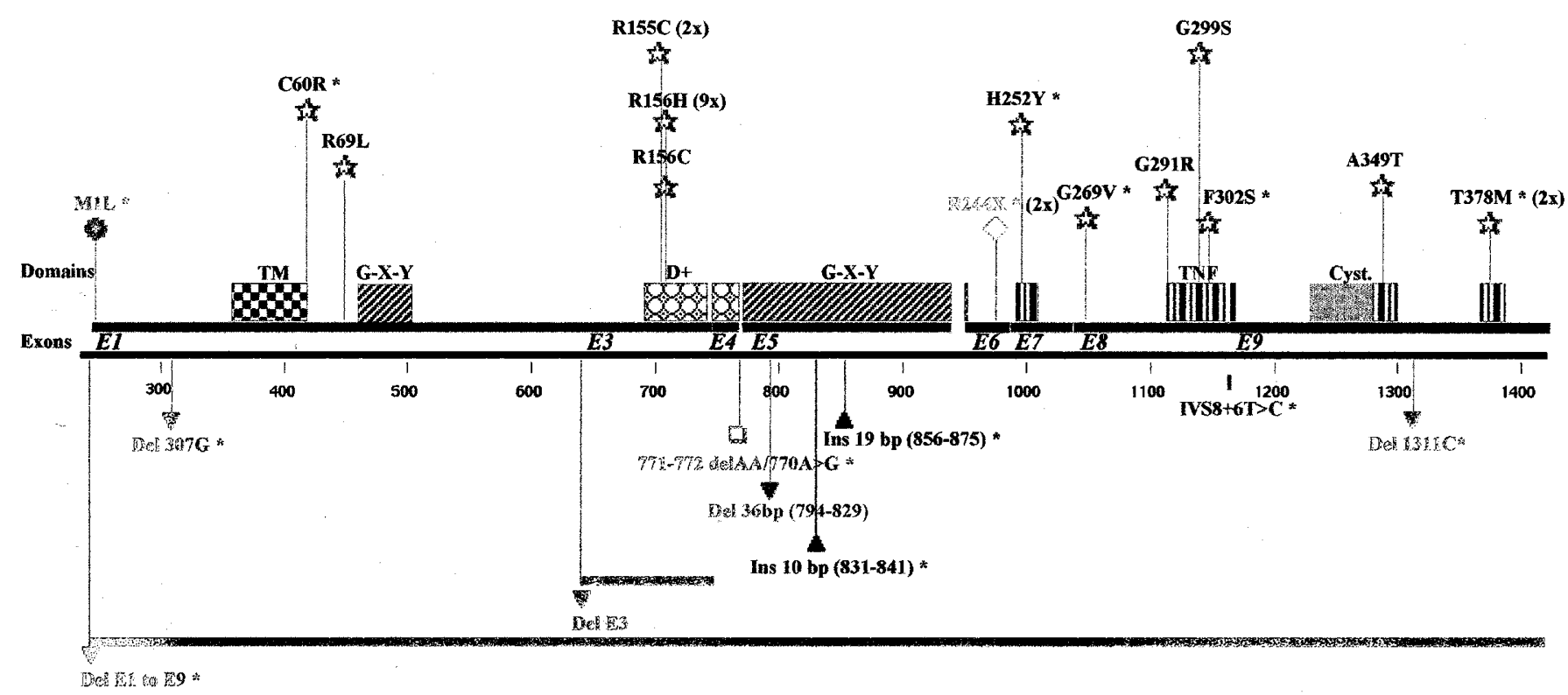

\footnotetext{
Figure 1 : Repartition of mutations in the ED1 gene from the present study

* = Novel mutations

$(2 x)=$ number in bracket indicates the number of occurrence reported for that mutation
}

ETM : Transmembrane domain $\mathbb{Z} \mathrm{G}-\mathrm{X}-\mathrm{Y}$ : Collagenous domain $\mathrm{B}$ D + : Positively charged domain

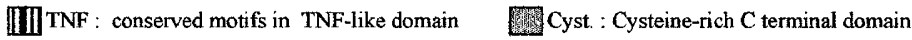

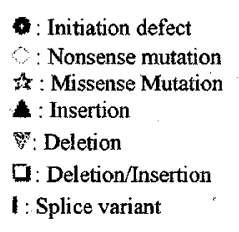

Figure 1 Distribution of mutations in the ED1 gene from the present study. Single nucleotide changes are represented above, and insertion/deletions below a schematic representation of the coding region that represents the various protein domains and the exons.

Mutations are distributed in all but exon 4 (and exon 2 that is present only in the shortest transcript). The novel missense mutations were not found on at least 80 normal chromosomes. One (C60R) affects the transmembrane domain while the four others (H252Y, G269V, F302S, T378M) map to the TNF-like domain (Figure 1). Three of them affect residues conserved in FAS ligand (H252, F302, T378). ${ }^{14}$ Notably, the histidine 252 residue is perfectly conserved in the TNF family members, and participates in the protomer's hydrophobic core required for trimer contacts. ${ }^{19}$ All of these missense mutations are chemically nonconservative, and affect residues that are conserved in the homologous murine Ta protein. In patient GT88/6327 the multiplex PCR suggested the presence of a large deletion encompassing all of the ED1 gene and the flanking markers DXS1689 and DXS 1690, indicating that the deletion is at least $2.6 \mathrm{Mb}$ long. ${ }^{11,20}$

We observed a splice site variant in the donor site of exon 8 $(1166+6 \mathrm{~T}>\mathrm{C}$ in family DK48/900). Although this variant was not found in more than 120 normal X-chromosomes, it is of uncertain pathogenic significance as the transcript analysis could not be performed. Though similar base changes at the same position have been observed to cause exon skipping or use of an alternative splice site in other monogenic diseases, ${ }^{21}$ we think that it is more likely to be a rare nonpathogenic variant because the score of the mutated splice signal is very similar ( 86 vs 84 , scoring program in http:// 125.itba.mi.cnr.it/genebin/wwwspliceview) to that of the wild donor splice site of exon 8 .

Few mutations were recurrent. While $\mathrm{R} 156 \mathrm{H}$ was found nine times, mutations R155C, R244X, T378M were found twice each in patients from distinct families. Haplotype analysis demonstrated that these mutations arose independently in each family (data not shown). In addition the most recurrent mutation $\mathrm{R} 156 \mathrm{H}$ occurred in a diseased boy, a symptomatic girl and in a carrier mother, as new events (patient EG59/3073, 629/1406 and CP53/1020 respectively). The mutations R155C, R156C and R156H in exon 3, which affect $\mathrm{CpG}$ sites, have been previously reported in four families (Table 1) and represent one third of mutations in our study.

In most cases (29/34) a familial study was possible allowing to trace the inheritance of the mutation. A de novo mutation was found in six families. Three times it appeared as a direct transmission from a non-carrier mother to an affected son. In the three remaining cases the mutation appeared de novo in carrier mothers. It originated once from a grand paternal 
Table 1 Nucleotide substitution in ED1 mutations spectrum

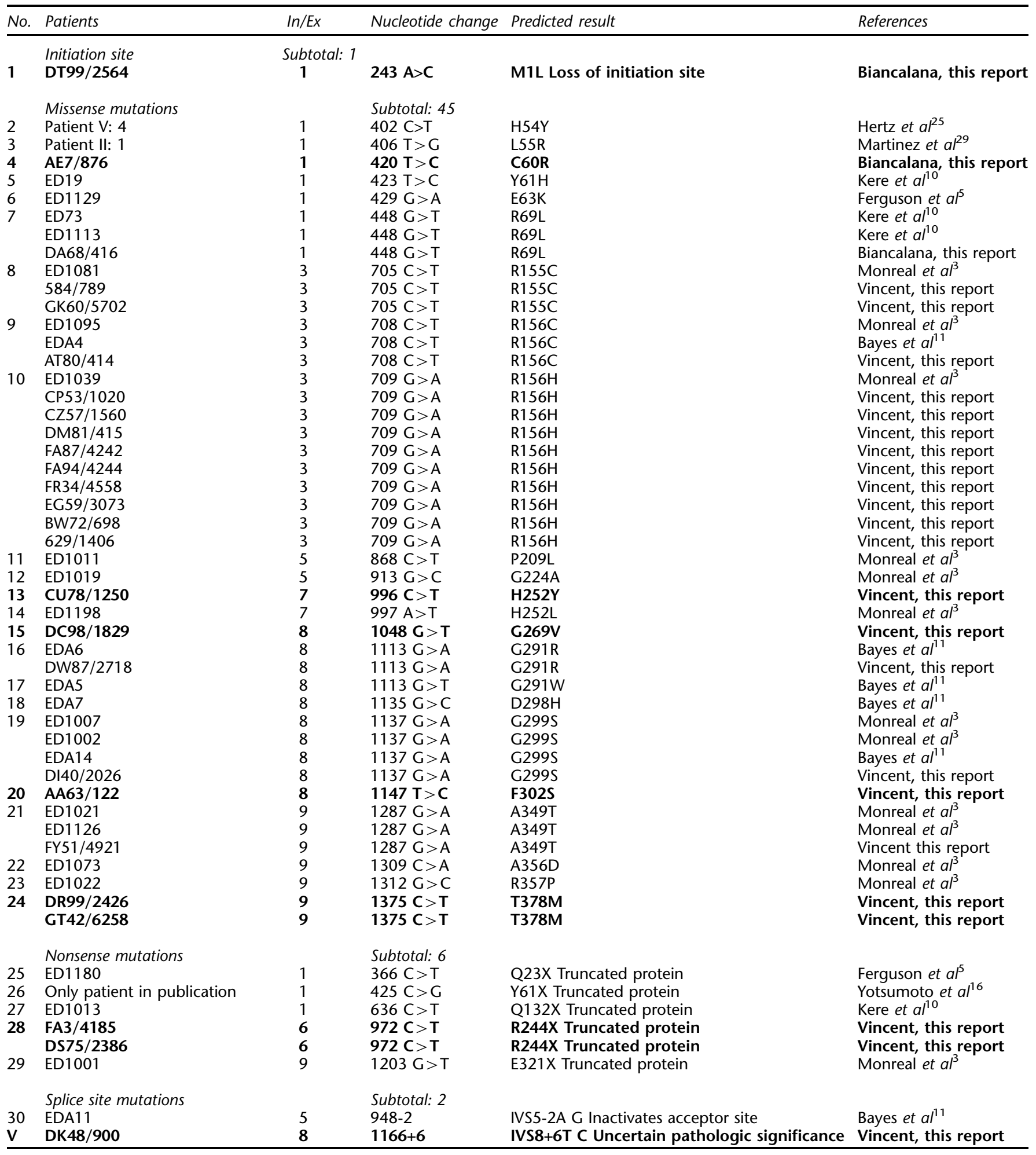

$\mathrm{V}$ : variant 
Table 2 Deletions/insertions ED1 mutations spectrum

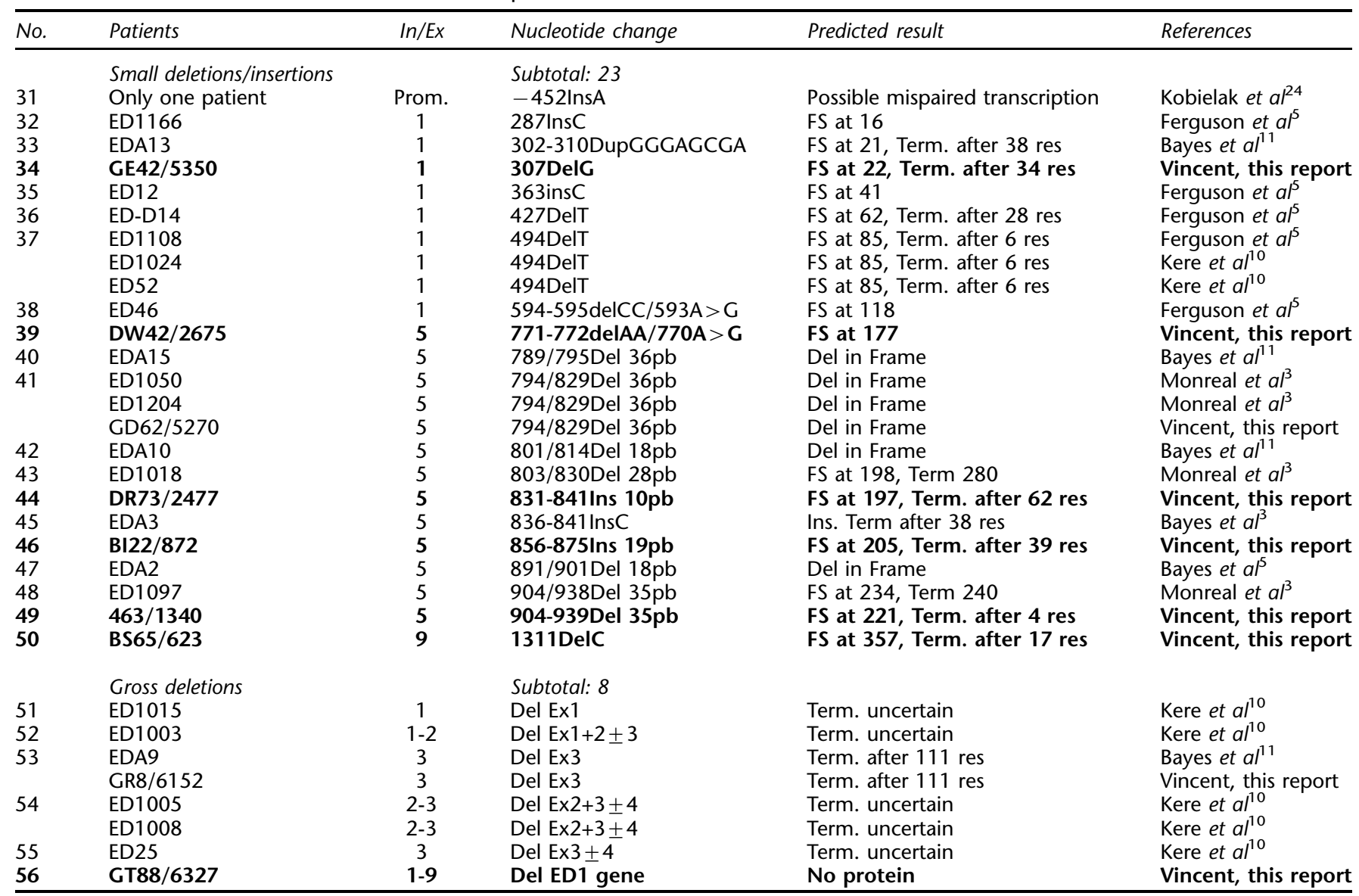

FS: frameshift, Term: premature termination, Res: residues.

sperm mutation but could not be traced in the two other cases.

\section{Genotype/phenotype correlation}

All affected males in our study had the classic XLHED phenotype. Besides the main signs, the patients displayed other features with varying degree. Review of clinical features showed no obvious phenotype/genotype correlation. We noticed an inter familial difference in expressivity in individuals bearing the same mutation (ie R156H) as well as intra familial variability in affected brothers. The nature of mutation did not appear to predict the severity of the disease. Notably, the large deletion encountered in patient GT88/ 6327 did not induce distinctive or more severe clinical manifestations, when compared with the ones associated to missense mutations (Table 3).

It is interesting to note that the two brothers with the initiation codon mutation (ATG to CTG) show a somewhat milder phenotype (with respect to sweat glands and hair development) than most other affected males in our series. As it has been reported that some translation can be initiated (albeit inefficiently in most cases) from a CTG codon, ${ }^{22,23}$ this suggests that residual synthesis of ectodysplasin occurred in these two patients.

Dental abnormalities (18/26), and mild hypotrichosis (14/ 24) were the most common signs encountered in female carriers in our study. Mild hypohidrosis (7/26) was less frequently present. This is in contrast with the frequent and severe sweat gland dysfunction in affected males. However heterozygous females may manifest patchiness of body sweating and other features, in addition to dental and scalp hair anomalies (Table 3 ). We examined the $\mathrm{X}$ inactivation pattern in leucocytes from 20 female carriers, including 17 with documented clinical record. A low (22-34\%) inactivation of the normal $\mathrm{X}$ chromosome was noticed in the four female carriers with minimal clinical expression of XLHED (Figure 2). Inactivation of the mutation-bearing $\mathrm{X}$ chromosome, as observed in white blood cells, did not however reflect the severity of the disease in female carriers with characteristic features of XLHED (Figure 2). 
Table 3 Review of clinical findings in our study

\begin{tabular}{|c|c|c|c|c|c|c|c|c|c|c|c|}
\hline \multirow{2}{*}{$\begin{array}{l}\text { Subject } \\
\text { Adn/family }\end{array}$} & \multirow[b]{2}{*}{ Sex } & \multicolumn{3}{|c|}{ Mutations } & \multirow[b]{2}{*}{ Abn Dev sweat } & \multirow[b]{2}{*}{ Abn Dev hair } & \multicolumn{2}{|c|}{ Clinical Features } & \multirow[b]{2}{*}{ Facies } & \multirow[b]{2}{*}{ Infections } & \multirow[b]{2}{*}{ Other } \\
\hline & & $I D$ & $1 / E$ & NI\% & & & Abn Dev teeth & Skin & & & \\
\hline \multicolumn{12}{|c|}{ Truncating mutations } \\
\hline DT99/2564 & $M$ & M1L & 1 & & +++ & +++ & +++ & +++ & - & + & \\
\hline DU2/2564 & M & M1L & 1 & & +++ & +++ & ++++ & +++ & - & + & $\mathrm{Ke}+\mathrm{Af}$ \\
\hline BI22/872 & M & Ins19pb & 5 & & ++++ & +++++ & - & ++ & + & ++ & Otitis \\
\hline DR73/2477 & M & Ins10pb & 5 & & ++++++ & ++++ & +++ & +++ & - & - & \\
\hline $463 / 1340$ & M & Del35pb & 5 & & ++++ & ++++ & +++ & +++ & +++ & - & \\
\hline GD62/5270 & M & Del36pb & 5 & & +++ & ++++ & +++ & + & ++ & - & \\
\hline BS65/623 & M & 1311delC & 9 & & +++++++ & +++ & +++++ & +++ & - & - & \\
\hline GT88/6327 & M & del & 9 & & ++++++ & ++++ & +++++ & ++ & ++ & - & As \\
\hline
\end{tabular}

Missense mutations

\begin{tabular}{|c|c|c|}
\hline AE7/139 & M & C60R \\
\hline $584 / 789$ & M & R155C \\
\hline GK60/5702 & M & R155C \\
\hline AT80/414 & M & R156C \\
\hline CP53/1020 & M & R156H \\
\hline CZ57/1560 & M & $\mathrm{R} 156 \mathrm{H}$ \\
\hline DM81/415 & M & R156H \\
\hline FA87/4242 & M & R156H \\
\hline FA94/4244 & $M$ & R156H \\
\hline FR34/4558 & M & $\mathrm{R} 156 \mathrm{H}$ \\
\hline EG59/3073 & $M$ & $\mathrm{R} 156 \mathrm{H}$ \\
\hline CU78/1250 & M & $\mathrm{H} 252 \mathrm{Y}$ \\
\hline AA63/122 & M & F302S \\
\hline AA69/122 & $M$ & F302S \\
\hline DR99/2426 & M & T378M \\
\hline GT42/6258 & M & T378M \\
\hline
\end{tabular}

DU1/2564 F $\quad$ M1L

298/789

GK49/5702

GK50/5702

AT79/414

AT82/414

DI45/414

CP52/1020

CP51/1020

CZ59/1560

DM82/415

DM80/415

FA85/4242

FN30/4244

FN29/4244

$629 / 1406$

GD61/5270

FA2/4185

DS75/2386

DO79/2386

CU81/1250

CU77/1250

AA65/122

AA50/122

AA52/122

DC98/1829

DS3/2426
R155C

R155C

R155C

R156C

R156C

R156C

$\mathrm{R} 156 \mathrm{H}$

$\mathrm{R} 156 \mathrm{H}$

$\mathrm{R} 156 \mathrm{H}$

$\mathrm{R} 156 \mathrm{H}$

$\mathrm{R} 156 \mathrm{H}$

$\mathrm{R} 156 \mathrm{H}$

$\mathrm{R} 156 \mathrm{H}$

$\mathrm{R} 156 \mathrm{H}$

$\mathrm{R} 156 \mathrm{H}$

Del36pb

R244X

R244X

R244X

$\mathrm{H} 252 \mathrm{Y}$

$\mathrm{H} 252 \mathrm{Y}$

F302S

$\mathrm{F} 302 \mathrm{~S}$

F302S

G269V

T378M

$\begin{array}{cc}+++ & +++ \\ +++++++ & +++ \\ +++++++ & +++ \\ +++++ & +++ \\ +++++ & +++++ \\ +++++ & +++++ \\ +++++ & +++ \\ +++++++ & +++ \\ +++ & +++ \\ ++++++ & +++ \\ ++++ & +++++ \\ ++++ & ++++ \\ +++++ & ++++ \\ +++++ & ++++ \\ ++++++ & +++++ \\ ++++++ & ++++\end{array}$

$\pm$

$-$

$-$

$-$

$+$

+++
-

$-$

++++

$-$

$-$

$+++$

$+++$

$-$

+++++

$-$

+++

$-$

$-$

ND

$++++$

$+++$

$+++$

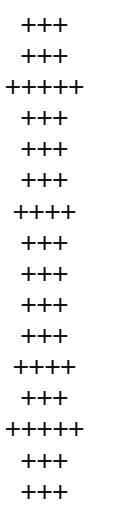

-
+
+++
+++
+++

+++
++

++
+
+

$\begin{array}{ccc}+ & + \\ ++ & ++\end{array}$

$\begin{array}{ll}++ & + \\ ++ & ++\end{array}$

$\begin{array}{lll}++ & ++ \\ +++ & ++\end{array}$

$+++$

$+\quad++$

++
++

$+$

MR

Icthyosis

As

$\mathrm{FPb}$

MR

$+++\quad+$

+++
-
+++
+++
+++
+++
+++
+++
-
+++
+++
+++
-
-
-
+++
+++
+++
+++
+++
+
+++
+++
+++
+++
+++
+

++
+
+

+
+
+
+
+
+
-
+
+
++
+
+
+

-
-
-
-
+
+
-
-
+
+
+
+
-
-
-
-

As

MA

$M A$

$\mathrm{MA}+\mathrm{FPb}$

As

$\mathrm{ND}$
+++

Abn: abnormal, Dev: development, As: asthma, Ke: keratitis, AF: allergy field, MA: mammary agenesia, FPb, feeding problem, MR: mental retardatation

$\mathrm{ND}=$ not determined (information or sample not available), $\mathrm{Nl}=$ not informative.

Anhidrosis with heat intolerance and pyrexia episodic $=+++++++$, anhidrosis with pyrexia episodic or heat intolerance $=++++++$, anhidrosis $=+++++$, hypohidrosis with heat intolerance and pyrexia episodic $=+++++$, hypohidrosis with pyrexia episodic or heat intolerance $=++++$, hypohidrosis $=+++$, heat intolerance $=+$; hypotrichosis $=+++$, hypotrichosis (scalp hair and eyebrow) $=++++$, hypotrichosis and no pilosity=+++++; anodontia =+++++, anodontia and hypodontia=++++, hypodontia=+++; dry skin and atopic ezcema=+++, atopic ezcema=++, dry skin=+; facial dysmorphic=one+per characteristic sign; susceptibility for infection=+, recurrent infections=++. 
III

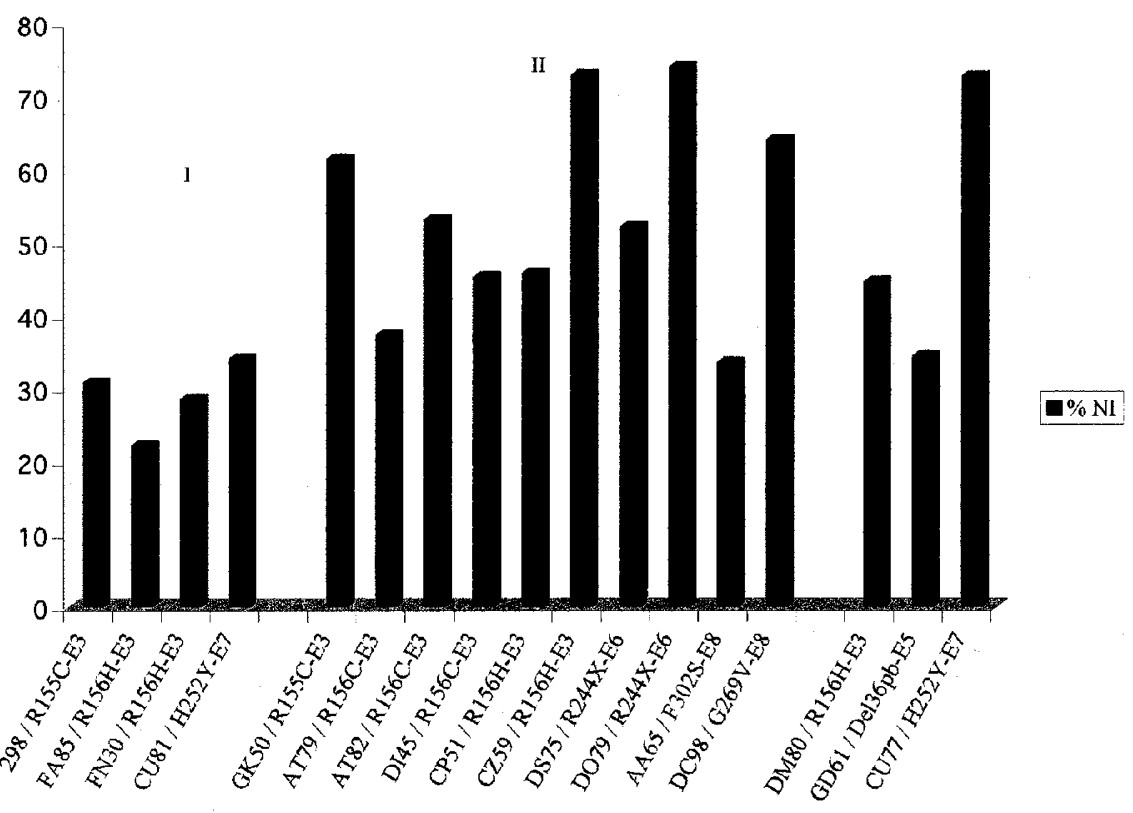

$\% \mathrm{NI}=$ percent of normal $X$ chromosome inactivation

$\mathrm{I}=$ no sign or no main sign

II = one or two main sign (Abnormal developpment of sweat gland, or teeth or hair)

III = at least three main signs (similar pherotype as the one described in affected male)

Figure $2 \mathrm{X}$ inactivation pattern in XHLED female carriers. Carriers informative for the analysis of X interaction at the androgen receptor locus were subdivided in three groups, according to the expression of the clinical features: I=no sign or no main sign; II=one of two main sign (abnormal development of the sweat gland, or teeth or hair); III=at least three main signs (similar phenotype as the one described in affected male). The per cent of normal $X$ chromosome inactivation is indicated in ordinates.

\section{Discussion}

Mutations spectrum and diagnostic implications

This is hitherto the most important series of ED1 mutations reported. We screened 52 unrelated families or sporadic cases, over all the coding sequence of the ED1 gene and we report 14 novel mutations. In families including several generations with affected subjects, we identified mutations in ED1 gene in $29 / 38$ (76\%) cases. No mutation was found in the four families with two affected sibs in one generation. In addition only 5/10 sporadic cases bore a mutated ED1 allele. This suggests that some cases may have an autosomal form such as the one generated by the DL gene anomalies. ${ }^{13}$

The $76 \%$ detection rate is slightly lower than expected considering previously reported detection rates in the two previous studies that analysed all exons (29 mutations in 33 families). ${ }^{6,11}$ A mutation remained undetected in several familial cases. As we focused our study on protein coding exonic regions and their flanking sequences, this suggests that mutations occur in non coding regions. To date only one such putative mutation was reported, in the promoter region. ${ }^{24}$ Missing mutations might lie in introns (notably in the $300 \mathrm{~kb}$ large intron that separates exon 1 from exon 3) or may correspond to gene rearrangements (inversions or duplications) that would not be detected by our approach.
Our findings, together with the published data (see Human Gene Mutation Database: www.uwcm.ac.uk) document ED1 mutations in 85 independent index cases (Tables 1 and 2). They correspond to 48 different point mutations (including small deletions or insertions), and eight larger deletions, including the complete gene deletion reported here. It is thus now possible to derive general conclusions regarding the type and location of mutations. The extensive mutation heterogeneity is expected for a severe X-linked disease that affects reproductive fitness (in this case, either by childhood lethality due to hyperthermia, aesthetic prejudice, or as a result of genetic counselling). Sixty-eight per cent of the 77 point mutations were found in exons 1,3 and 5 . However the frequency of mutations in exon $1(21 / 77)$ is probably biased upwards by the initial studies that only analysed this exon, ${ }^{5,10}$ and mutations in this exon appear to account for about $5-10 \%$ of patients (four of the 34 identified mutations in our series and $\mathrm{in}^{5,10}$ ). Thus, this exon shows lower density of mutations than expected from its size (one third of the coding sequence), and from its richness in CpG dinucleotides (61\% of the coding sequence's CpGs ).

A striking hot spot of mutations is constituted by the CpG rich sequence in exon 3 , at arginine codons 155 and 156 (CGC CGC), as 16 families carry one of the three missense 
mutations (R155C, R156C or R156H), that also point out the functional importance of these residues. The pattern is very different in exon 5 , as most of the mutations (11 of 15) are deletions or insertions of 10 to $36 \mathrm{bp}$, six of them in frame. These occur in the Gly-X-Y collagen like domain, and it is likely that the repetitive nature of this region favours this type of mutations, deriving from polymerase slippage. All 10 mutations detected in exon 8 are missense mutations affecting the TNF-like domain. No mutations have been observed up to now in exon 2 (that encodes only three amino-acids of the short ectodysplasin isoform), and in the very small ( $23 \mathrm{bp}$ ) exon 4 . These data thus indicate that exons 1,3 and 5 should be tested first, followed by exons 8 and 9 , for an efficient mutation screening.

\section{Genotype-phenotype correlations}

Mutation detection has already proven to be very useful for genetic counselling, carrier detection and prenatal diagnosis in XLHED families. However, there is no obvious correlation between the type of mutation and the severity of the disease, as also reported in previous studies. ${ }^{5,6}$ Inter- and intrafamilial variability of clinical expression in male patients was noticed in our series as in a previous report. ${ }^{25}$

XLHED shows partial penetrance in female carriers ${ }^{12,26,27}$ and the probability to detect them by clinical examination was estimated as being $60-70 \%{ }^{28}$ This should be reappraised with regard to the contribution of molecular analysis. Indeed in our series, only two proven female carriers (8\%) demonstrated no clinical sign among the 26 examined within the 34 families with an identified mutation.

The reduced penetrance in female carriers can be explained by random $\mathrm{X}$ chromosome inactivation. As ectodysplasin is most likely a cell autonomous protein, having an intracellular domain, female carriers are mosaics of functionally normal and abnormal cells, and clinical findings are likely to depend on the percentage of abnormal cells in the progenitor cells for sweat glands, hair and teeth. Although our study of $\mathrm{X}$ inactivation was limited by the number of available and informative (at the androgen receptor test locus) carriers, one can conclude that there is little correlation between the pattern of $\mathrm{X}$ inactivation observed in leucocytes and the severity of clinical expression, in contrast with a suggestion by Martinez et al. ${ }^{29}$ However, the four informative carriers who showed no or minimal clinical signs, had the lowest level of inactivation of the normal $\mathrm{X}$ chromosome, in leucocytes. This suggests that general biased inactivation (that might in some cases results from selection at another $\mathrm{X}$ chromosome locus, or from polymorphism at a locus controlling inactivation, such as Xist) may modulate phenotypic expression in some carriers.

\section{Acknowledgements}

We wish to thank Michèle Schmitt and Stéphanie Despiau for their technical assistance in the sequencing work. We would also like to thank all clinicians for blood samples of XLHED families and phenotypic data. This study was supported by the Centre Hospitalier Universitaire Régional of Strasbourg and the Centre Hospitalier Universitaire of Toulouse.

\section{References}

1 Clarke A: Hypohidrotic ectodermal dysplasia. J Med Genet 1987; 24: $659-663$.

2 Park JW, Hwang JY, Lee SY, Lee JS, Go MK, Whang KU: A case of hypohidrotic ectodermal dysplasia. J Dermatol 1999; 26: 44-47.

3 Monreal A, Zonana J, Ferguson B: Identification of a new splice form of the ED1 gene permits detection of nearly all X-linked hypohidrotic ectodermal dysplasia mutations. Am J Hum Genet 1998; 63: 380-389.

4 Pinheiro M, Freire-Maia N: Ectodermal dysplasias: A clinical classification and a causal review. Am J Med Genet 1994; 53 $153-162$.

5 Ferguson B, Thomas N, Munoz F, Morgan D, Clarke A, Zonana J: Scarcity of mutations detected in families with $\mathrm{X}$ linked hypohidrotic ectodermal dysplasia: diagnostic implications. $J$ Med Genet 1998; 35: 112-115.

6 Zonana J, Gault J, Jones M et al: Detection of a molecular deletion at the DXS732 locus in a patient with X-linked hypohidrotic ectodermal dysplasia (EDA), with the identification of a unique junctional fragment. Am J Hum Genet 1993; 52: $78-84$.

7 Clarke A, Sarfarazi M, Thomas NST, Roberts K, Harper PS: XLinked hypohidrotic ectodermal dysplasia: DNA probe linkage analysis and gene localization. Hum Genet 1987; 75: 378 - 380.

8 Zonana J, Jones M, Browne D et al: High resolution mapping of the X-linked hypohidrotic ectodermal dysplasia (EDA) locus. Am J Hum Genet 1992; 51: 1036-1046.

9 Srivastava A, Montonen O, Saarialho-Kere U et al: Fine mapping of the EDA gene: a translocation breakpoint is associated with a CpG island that is transcribed. Am J Hum Genet 1996; 58: $126-$ 132.

10 Kere J, Srivastava A, Montonen O et al: X-linked anhidrotic (hypohidrotic) ectodermal dysplasia is caused by mutation in a novel transmembrane protein. Nature Genet 1996; 13: 409-416.

11 Bayes M, Hartung A, Ezer S et al: The anhidrotic ectodermal dysplasia gene (EDA) undergoes alternative splicing and encodes ectodysplasin A with deletion mutations in collagenous repeats. Hum Mol Genet 1998; 7: 1661-1669.

12 Munoz F, Lestringant G, Sybert V et al: Defenitive evidence for an autosomal recessive form of hypohidrotic ectodermal dysplasia clinically indistinguishable from the more common X-linked disorder. Am J Hum Genet 1997; 61: 94-100.

13 Monreal A, Ferguson B, Headon D, Street S, Overbeek P, Zonana $\mathrm{J}$ : Mutations in the human homologue of mouse dl cause autosomal recessive and dominant hypohidrotic ectodermal dysplasia. Nature Genet 1999; 22: 366-369.

14 Ezer S, Bayes M, Elomaa O, Schlessinger D, Kere J: Ectodysplasin is a collagenous trimeric type II membrane protein with a tumor necrosis factor like domain and co-localizes with cytoskeletal structures at lateral and apical surfaces of cells. Hum Mol Genet 1999; 8: 2079-2086.

15 Ezer S, Schlessinger D, Srivastava A, Kere J: Anhidrotic ectodermal dysplasia (EDA) protein expressed in MCF-7 cells associates with cell membrane and induces rounding. Hum Mol Genet 1997; 6: $1581-1587$

16 Yotsumoto S, Fukumaru S, Matsushita $S$ et al: A novel point mutation of the EDA gene in a japanese family with anhidrotic ectodermal dysplasia. J Invest Dermatol 1998; 111: 1246-1247.

17 Budowle B, Chakraborty R, Giusti A, Eisenberg A, Allen C: Analysis of the VNTR locus D1S80 by the PCR followed by highresolution PAGE. Am J Hum Genet 1991; 48: 137-144. 
18 Allen RC, Zoghbi H, Moseley A, Rosenblatt H, Belmont JW: Methylation of HpaII and HhaI sites near the polymorphic CAG repeat in the human androgen-receptor gene correlates with $\mathrm{X}$ chromosome inactivation. Am J Hum Genet 1992; 51: 1229 1239.

19 Mikkola M, Pispa J, Pekkanen M et al: Ectodysplasin, a protein required for epithelium morphogenesis, is a novel TNF homologue and promotes cell-matrix adhesion. Mech Dev 1999; 88: $133-146$.

20 Week DE, Nygaard TG, Neystat M, Harby LD, Wilhelmsen KC: A high-resolution genetic linkage map of the pericentromeric region of the human X chromosome. Genomics 1995; 26: 39 46.

21 Krawczak M, Reiss J, Cooper D: The mutational spectrum of a single base-pair substitutions in mRNA splice junctions of human genes: causes and consequences. Hum Genet 1992; 90 $41-54$.

22 Carroll R, Derse D: Translation of equine infectious anemia virus bicistronic tat-rev mRNA requires leaky ribosome scanning of the tat CTG initiation codon. J Virol 1993; 67: 1433-1440.

23 Takayama S, Krajewski S, Krajewska $M$ et al: Expression and location of Hsp70/Hsc-binding anti-apoptotic protein BAG-1 and its variants in normal tissues and tumor cell lines. Cancer Res 1998; 58: 3116-3131.
24 Kobielak K, Kobielak A, Limon J, Trzeciak: Mutation in the regulatory region of the EDA gene coincides with the symptoms of anhidrotic ectodermal dysplasia. Acta Biochim Pol 1998; 45: $245-250$.

25 Hertz JM, Norgaard Hansen K, Juncker I, Kjeldsen M, Gregersen $\mathrm{N}$ : A novel missense mutation $(402 \mathrm{C} \rightarrow \mathrm{T})$ in exon 1 in the EDA gene in a family with X-linked hypohidrotic ectodermal dysplasia. Clin Genet 1998; 53: $205-209$.

26 Gilgenkrantz S, Blanchet-Bardon C, Nazzaro V, Formiga L, Mujica P, Alembik Y: Hypohidrotic ectodermal dysplasia: clinical study of a family of 30 over three generations. Hum Genet 1989; 81: 120-122.

27 Cambiaghi S, Restano L, Paakkonen K, Caputo R, Kere J: Clinical finding in mosaic carriers of hypohidrotic ectodermal dysplasia. Arch Dermatol 2000; 136: $217-224$.

28 Pinheiro M, Ideriha MT, Chautard-Freire-Maia EA, Freire-Maia N, Primo-Parmo SL: Christ-Siemens-Touraine syndrome. Investigations on two large brazilian kindreds with a new estimate of the manifestation rate among carriers. Hum Genet 1981; 57: 428-431.

29 Martinez F, Millan JM, Orellana C, Prieto F: X-linked anhidrotic (hypohidrotic) ectodermal dysplasia caused by a novel mutation in ED1 dene 406T > G (Leu55Arg). J Invest Dermatol 1999; 113: $285-286$. 